\title{
Underuse of spirometry in the diagnosis of COPD
}

Caramori reports on the underuse of spirometry in the diagnosis of COPD by Italian general practitioners (GPs) [1]. Lack of access to lung function laboratories and the belief that spirometry is not necessary for the diagnosis of COPD were the main barriers. The authors suggest special educational programmes to correct this deficiency.

In line with other studies, spirometric confirmation of COPD diagnosis was low among Italian GPs [2, 3]. Implementation of spirometry could indeed be enhanced by disposing of several barriers that impede GPs in the use of spirometry. Essential the first step is to aim to obtain the facilities so that quality spirometry can be widely available and easily accessible. This could be either by means of centralised services like primary care group commissioned services, by arranging open access to pulmonary function laboratories, in the practice itself or through a combination of these options.

Even with good accessibility of services there is no guarantee that GPs will indeed use spirometry in all cases. Carrying out spirometry in general practice seems indeed justified in terms of test validity, provided that practice staff have been trained sufficiently [4]. This creates an essential precondition for implementation of spirometry in the general practice setting.

Little is known about the reasons why GPs choose to use spirometry or to refrain from it, but in a recent survey we identified a number of GPrelated and practice-related factors that could play a role [5]. The capability to organise spirometry, the possibility of delegation of tasks by GPs to practice assistants or practice nurses, the guaranteed availability of equipment on every occasion that is needed are all practice-related factors which are likely to be involved [5].
Lack of adequate training in the use and interpretation seem to be the most important GP-related factors [2].

Once a GP is convinced that spirometry adds relevant information in the assessment of a COPD diagnosis, on-going special educational programmes that focus in particular on the interpretation of spirometric tests could take away GPs' lack of self-confidence with respect to this topic.

We believe that practice-related factors (e.g. presence of a practice nurse, delegation of medical tasks to the practice assistant) are particularly crucial in the improvement of actual integration of spirometry into the GP's management of respiratory diseases.

P.J.P. Poels, T.R.J. Schermer, C. van Weel

Correspondence: P.J.P. Poels MD, Department of General Practice (117); Radboud University Nijmegen Medical Centre; P.O. Box 9101; 6500 HB Nijmegen; The Netherlands; e-mail: p.j.p.poels@hag.umcn.nl

\section{References}

1. Caramori G, Bettoncelli G, Tosatto R, et al. Underuse of spirometry by general practitioners for the diagnosis of COPD in Italy. Monaldi Arch Chest Dis 2005; 63: 6-12.

2. Bolton $\mathrm{CE}$, Ionescu AA, Edwards $\mathrm{PH}$, et al. Attaining a correct diagnosis of COPD in general practice. Respir Med 2005; 99: 493-500.

3. Decramer M, Bartsch P, Pauwels R, et al. Management of COPD according to guidelines. A national survey among Belgian physicians. Monaldi Arch Chest Dis 2003; 59: 62-80.

4. Schermer TR, Jacobs JE, Chavannes NH, et al. Validity of spirometric testing in a general practice population of patients with chronic obstructive pulmonary disease (COPD). Thorax 2003; 58: 861-866.

5. Poels PJP, Schermer TRJ, Jacobs JE et al. Determinants for implementation of spirometry by Dutch general practitioners. Eur Respir J 2004; 24 (Suppl 48): 472s.

\section{The authors' reply}

We agree in general with the comments made by Poels and colleagues that practice-related factors are essential to increase the use of spirometry by general practitioners (GPs). However, there are two major problems that mitigate against an increase in the use of spirometry in general practice in Italy: a) understanding that spirometry is the gold standard for the diagnosis and management of COPD b) easily accessible spirometry facilities when required. In fact most patients with GOLD stage 3 and 4 COPD have undergone spirometry because they are treated collaboratively with respirologists. At the opposite end of the scale of severity GOLD stage 0,1 and 2 patients only rarely undergo spirometry. It appears that this exam is not considered important for the population at large in the same way that blood pressure and cholesterol measurements are for the prevention of cardiovascular disease. GPs still believe that promotion of measures to increase smoking cessation are enough. Hence this is a cultural prob- lem for general practice. It is very difficult to guarantee a qualitatively sufficient standard of spirometric testing to the population in question using the current organizational model in Italian general practice. This model is mainly composed of practices with a single doctor who works independently and without nurses. Under these circumstances general practitioners have a problem of time and maintenance of technical competency and equipment calibration. Recently thanks to interventions promoted by the Italian National Health Service, primary care structures are starting to emerge which allow physicians to work together (team medicine) and are generally equipped with paramedics. Within these structures, administrators focus on hiring/ training properly qualified internal personnel who have the ability to operate all the requisite mobile equipment in a satellite fashion. Hence it should be possible to increase the availability of spirometry to those subjects in need at the general practitioner's 
office. Currently there is also not enough evidence regarding telemedicine-assisted home spirometry to justify a long-term controlled trial in general practice [1-4].

G. Caramori1, G. Bettoncelli2, M. Adcock ${ }^{3}$, A. Papi ${ }^{1}$

1 Centro di Ricerca su Asma e BPCO, University of Ferrara,

2 Gruppo CORDIS on behalf of the Società Italiana di Medicina Generale (SIMG), Firenze, Italy.

3 Airway Diseases Section, NHLI, Imperial College London, London, UK.

Correspondence: Gaetano Caramori MD, PhD, Centro di Ricerca su Asma e BPCO, Via Savonarola 9, University of Ferrara, 44100 Ferrara, Italy; e-mail: crm@unife.it

\section{References}

1. Abboud S, Bruderman I. Assessment of a new transtelephonic portable spirometer. Thorax 1996; 51: 407-410.

2. Izbicki G, Abboud S. Telespirometry for home monitoring of pulmonary function. $J R$ Soc Med 1999; 92: 154-155.

3. Maree DM, Videler EA, Hallauer M, Pieper CH, Bolliger CT. Comparison of a new desktop spirometer (Diagnosa) with a laboratory spirometer. Respiration 2001; 68: 400-404.

4. Vontetsianos T, Giovas P, Katsaras T, et al. Telemedicine-assisted home support for patients with advanced chronic obstructive pulmonary disease: preliminary results after nine-month follow-up. J Telemed Telecare 2005; 11 (suppl 1): 86-88.

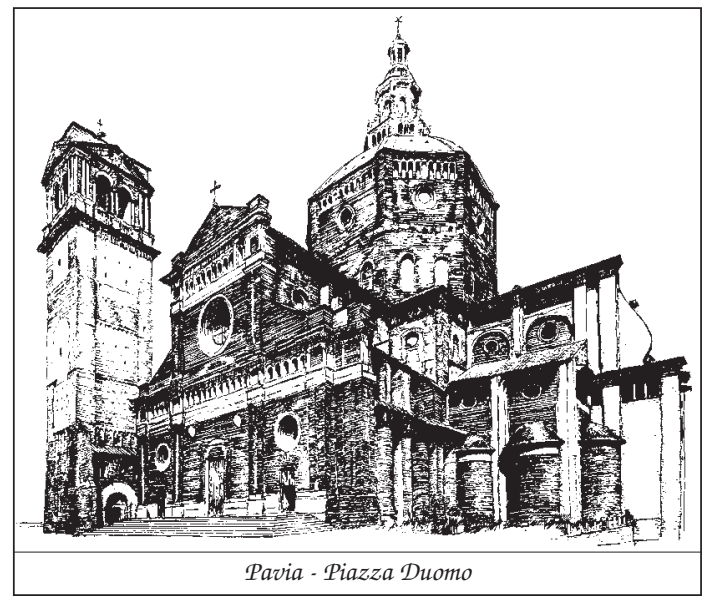

